\title{
The Effect of a Hybrid Method for Indoor Location
}

\author{
Hyeoncheol Zin ${ }^{2}$, HyunJin Park ${ }^{1}$, ChongGun Kim ${ }^{* 1}$ \\ ${ }^{1}$ Dept. of Computer Engineering, Yeungnam University, 280 Daehak-Ro, \\ Gyeongsan, Gyeongbuk 38541, Republic of Korea (38541 Korea) \\ ${ }^{2}$ SejoongIS CO, 831 Bon-dong, Dalseo-gu, Daegu, Republic of Korea.
}

\begin{abstract}
One of the popular methods studied for indoor positioning is based on Wi-F̂t sighal strength. Variation in signal strength that depends on communication distonge is an important parameter. But signal strength shows not only intuitive results but also irregular results. Depending on the experiment, using only wireless signal strength to determine communication distance may have evident positioning errors. Therefore, some additional methods for increasing positioning accuracy are needed. Time of flight (ToF) and additional sensors to support positioning are needed.In this study, a method based on only Wi-Fi signal strength and other hybrid methods which ane used additional upon wireless signal method are studied. Compass sensors and laser sensors were proven to be good additional methods for better positioning in a Wi-Fi-based system.
\end{abstract}

Keywords: Wi-Fi, RSSI, Indoor Position, Triangulation, Laser, Compass, Time of Flight

\section{Introduction}

The need for indeor Ocation tracking of mobile objects is increasing for a variety of applications, so various studies for focation tracking are ongoing $[9,10,16,18]$.

Methods for determining indoor position include $\mathrm{Wi}-\mathrm{Fi}$, radio frequency identification (RFID), Bluetooth, and ZigBee. The triangulation positioning method for indoor location based on Wi-Fisignal strength is used by selecting at least three access points (APs) based on received signal strengthy indication (RSSI) values, which reflect shorter distances. To increase positioning Correctness, the topology of the APs within an indoor area is also considered. To decrease positioning error based on only Wi-Fi RSSI, a laser sensor and a geomagnetic sensor are additionally used for precise locations in some environments.

\section{Previous and Related Studies}

Wi-Fi signal strength is used to find distances through the ChipconCO function. Some of the APs are arranged to measure indoor location using triangulation. Major methods of indoor location tracking are cell-ID, triangulation, and fingerprint [1]. Triangulation calculates the location of mobile terminals based on the distance from three known reference points. Comparing the mobile terminals' real-time signal patterns with a previously tracked signal pattern database is called the fingerprint method [1] to determine a mobile terminal's location. This paper uses triangulation as a position measurement method. Due to the deficiency in distance measurement using only RSSI [2], solutions to this problem use a laser sensor and a geomagnetic sensor.

\subsection{A Method for Distance Prediction by Radio Signal Strength}

The Friis formula and the ChipconCO formula can be used for calculating distance between two objects (http://www.ti.com).

Although prediction and calculation of distance based on radio signal strength is 
thought to be straightforward, the results are complex depending on the environment. In particular, calculation error in a distance decision is a major problem that has to be solved. In this study, the ChipconCO formula is used for simplicity in distance calculations based on signal strength:

RSSI $=-\left(10 \log _{10} d+A\right)$

In Formula (1), RSSI is the signal strength, $\mathrm{n}$ is propagation loss, $\mathrm{d}$ is distance, and A is the signal strength at a $1 \mathrm{~m}$ distance from the signal transmitter. The distance between the receiving object and the signal transmitting AP can be calculated using the ChipconCO formula with the above parameters.

Signal strengths affected by the surrounding environment can vary depending on the time. Therefore, the distance from the receiving object to the AP is difficult to calculate precisely.

Even so, accurate determination of A and $\mathrm{n}$ in Formula (1) can increase the accuracy of the distance calculation based on RSSI [11].

2.2 The RSSI Signals Strengths depend on Different Distancês

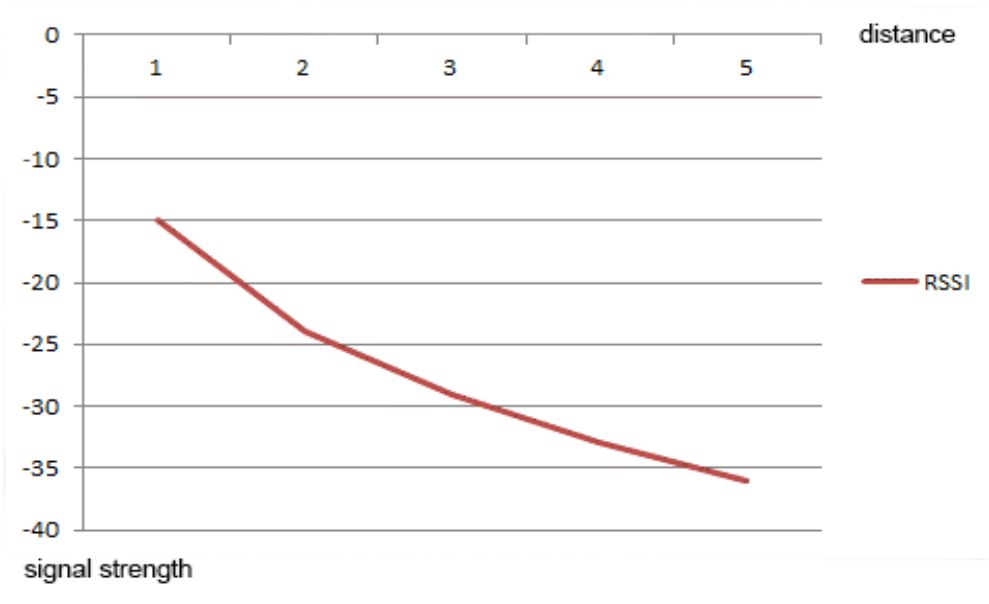

Figure 1. Change of the RSS1 Values Corresponding to the Distance between Transmitter and a Receiver

Figure 1 shows propagation loss $\mathrm{n}$ is 3.0 , and signal strength at $1 \mathrm{~m}$ from transmitter A is $15 \mathrm{dBm}$ from Formula (1).

Figure 1 shows that some RSSI values correspond to distance.

Significan phenomena shown in Figure 1 are as follows: in a $1 \mathrm{~m}$ to $3 \mathrm{~m}$ range, the signal strength apparently depends on distance, but the signal strength shows an insignificant change when the distance is greater than $4 \mathrm{~m}$.

\subsection{The Characteristics of RSSI Signal Strength}

Table 1 shows the signal strength from $1 \mathrm{~m}$ to $7 \mathrm{~m}$ at $1 \mathrm{~m}$ intervals which is the distance from the AP to the mobile object.

\section{Table 1. RSSI Values Corresponding to Distance}

\begin{tabular}{|c|c|}
\hline Distances & RSSI values \\
\hline $1 \mathrm{~m}$ & $-16.5 \mathrm{dBm}$ \\
\hline $2 \mathrm{~m}$ & $-19.2 \mathrm{dBm}$ \\
\hline $3 \mathrm{~m}$ & $-24.0 \mathrm{dBm}$ \\
\hline $4 \mathrm{~m}$ & $-28.8 \mathrm{dBm}$ \\
\hline
\end{tabular}




\begin{tabular}{|l|l|}
\hline $5 \mathrm{~m}$ & $-31.5 \mathrm{dBm}$ \\
\hline $6 \mathrm{~m}$ & $-31.9 \mathrm{dBm}$ \\
\hline $7 \mathrm{~m}$ & $-31.7 \mathrm{dBm}$ \\
\hline
\end{tabular}

In the $1 \mathrm{~m}$ to $5 \mathrm{~m}$ range, the difference in the signal strength can be recognized. At $1 \mathrm{~m}$ to $3 \mathrm{~m}$, the calculated distance using RSSI signal strength is similar to the actual distance [5], and signal strength from $4 \mathrm{~m}$ to $5 \mathrm{~m}$ shows relatively insignificant change. In distances farther than $6 \mathrm{~m}$, there is almost no change in signal strength for several distances [8]. Therefore, we decide that a meaningful distance is within $5 \mathrm{~m}$ when calculating distance from RSSI signal strength.

Radio signals used by wireless communications have transmission losses, and example causes of transmission loss are as follows [8]:

-an increase in distance in a free zone

-raindrops scattering radio waves

-the diffraction of radio waves

Table 2. RSSI Signal Samples at a 2 m Distance between Transmitter and Recelver

\begin{tabular}{|c|c|}
\hline RSSI values & Numbers \\
\hline$-18 \mathrm{dBm}$ & 23 \\
\hline$-19 \mathrm{dBm}$ & 17 \\
\hline$-20 \mathrm{dBm}$ & 2 \\
\hline$-21 \mathrm{dBm}$ & 1 \\
\hline$-50 \mathrm{dBm}$ below & 7 \\
\hline
\end{tabular}

Table 2 shows the colfected data for RSSI signals from an AP that used a $5 \mathrm{GHz}$ bandwidth 50 times. Even when the same devices were used for the experiments, the data at a $2 \mathrm{~m}$ distance between transmitter and receiver showed various RSSI values, depending on time. As shown in Table 2, some weak signals below $-50 \mathrm{dBm}$ were occasionally obtained in an actuál test.

If we use the average of the 50 sample data as the representative value, without filtering, then some abnormal values can have serious effects. And an abnormal value will cause a large errorin positioning. So, the median value is used from the sample data to solve this problen [1.

\subsection{Compass Sensing}

A compass sensor is a digital compass that measures the Earth's magnetic field.

It measures distance using a laser sensor when the sensor indicates the direction to any wall based on the room layout [15].

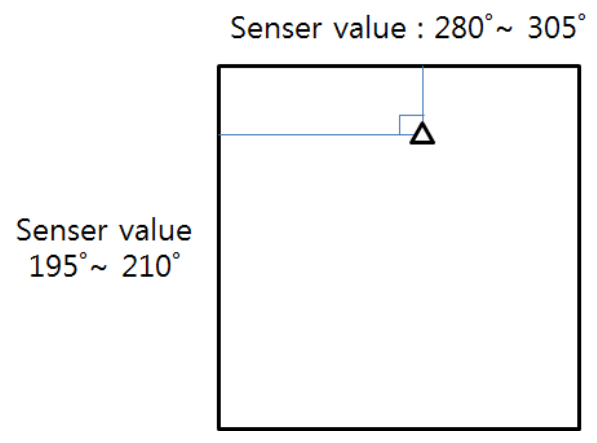

Figure 2. Target Wall 
Figure 2 shows the direction values from the two walls.

The position of the triangle in Figure 2 was measured using direction information from mapping the room layout. By positioning a laser sensor perpendicular to the wall using compass sensor information, the correct distance can be obtained.

Because a compass sensor obtains a wide range of geomagnetic values, the accuracy of the compass sensor is determined by correctly evaluating the magnetic field.

A magnetic field is the magnetic effect of electric current and magnetic material. A magnetic field is influenced by metal, and the magnetic field of the Earth's surface is 25 $65 \mathrm{uT}$.

If the magnetic field value at a position is not $25 \sim 65 \mathrm{uT}$, the position is under the influence of the metal.

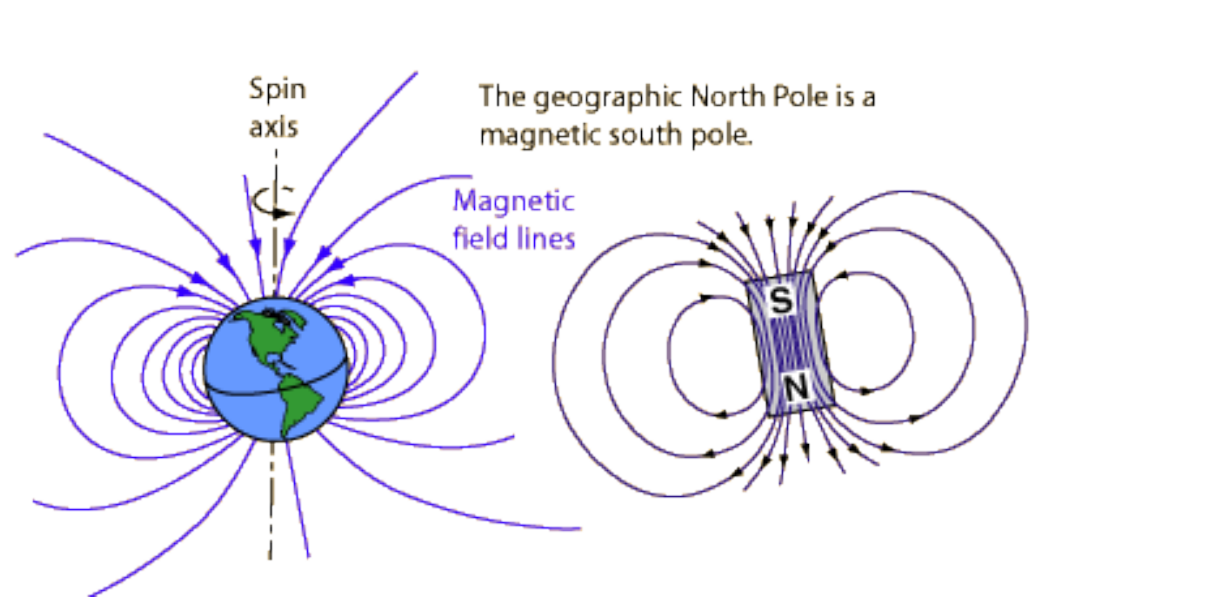

Figure 3. Earth's Magnetic Field

Figure 3 shows the generation prinerple of the Earth's magnetic field. The cause of the Earth's magnetic field is described by the dynamo theory whereby magnetic material is formed over the whole,Earth, generating an electric current within the Earth [19].

\subsection{Laser Selnsor}

A laser sensor calculates distance using the time taken to send and receive a laser beam between the transmitter and the receiver. The position and orientation of the walls are very important for esfimating the position of a mobile robot in [4]. To obtain the wall parameters, a predefined room layout is one solution.

\section{Table 3. The Laser Sensor Measures the Distance Error Rate}

\begin{tabular}{|l|l|l|l|l|l|}
\hline $\begin{array}{l}\text { Actual } \\
\text { distance }\end{array}$ & $1 \mathrm{~m}$ & $2 \mathrm{~m}$ & $3 \mathrm{~m}$ & $4 \mathrm{~m}$ & $5 \mathrm{~m}$ \\
\hline $\begin{array}{l}\text { Measurement } \\
\text { distance }\end{array}$ & $1.01 \mathrm{~m}$ & $2.02 \mathrm{~m}$ & $3.02 \mathrm{~m}$ & $4.03 \mathrm{~m}$ & $5.05 \mathrm{~m}$ \\
\hline Error rate & $1 \mathrm{~cm}$ & $2 \mathrm{~cm}$ & $2 \mathrm{~cm}$ & $3 \mathrm{~cm}$ & $5 \mathrm{~cm}$ \\
\hline
\end{tabular}

Table 3 shows error rates when measuring distance using a laser sensor.

The results from the laser sensor show smaller distance errors.

\subsection{LoS Components from Multipath Components}

RSSI simply capture the total received power at the mobile object. Because of irregular signal attenuation is caused by multipath fading. To mitigate the effect of multipath fading, extract $\operatorname{LoS}$ (Line of sight) components from multipath components is needed. Signal 
components except the LoS components may be delivered with some reflection from wall, floor and/or other objects, therefore it is natural to consider the reflected components have relatively low amplitude. CSI(Channel State Information) contains information about the channel at the level of individual subcarriers. CSI consists of amplitude and phase per subcarriers. Initially, CSI value is a frequency domain representation. IFFT(Inverse Fast Fourier Transform) can invert frequency domain to time domain. By filtering out signal components which are lower than 50\% of peak amplitude and remained signal components are again changed to the frequency domain one with applying FFT(Fast Fourier Transform). The signal is used for distance estimation. It can decrease multipath effects [17].

\subsection{Time of Flight}

ToF is the time it takes for the wireless signal to travel between transmission and reception devices. ToF uses the channel status information (CSI) provided by the communication devices under WiFi.

The CSI is measured at all of 64 different subcarriers, it is nore advantageous for distance measurement than RSSI represented by a singleincex.

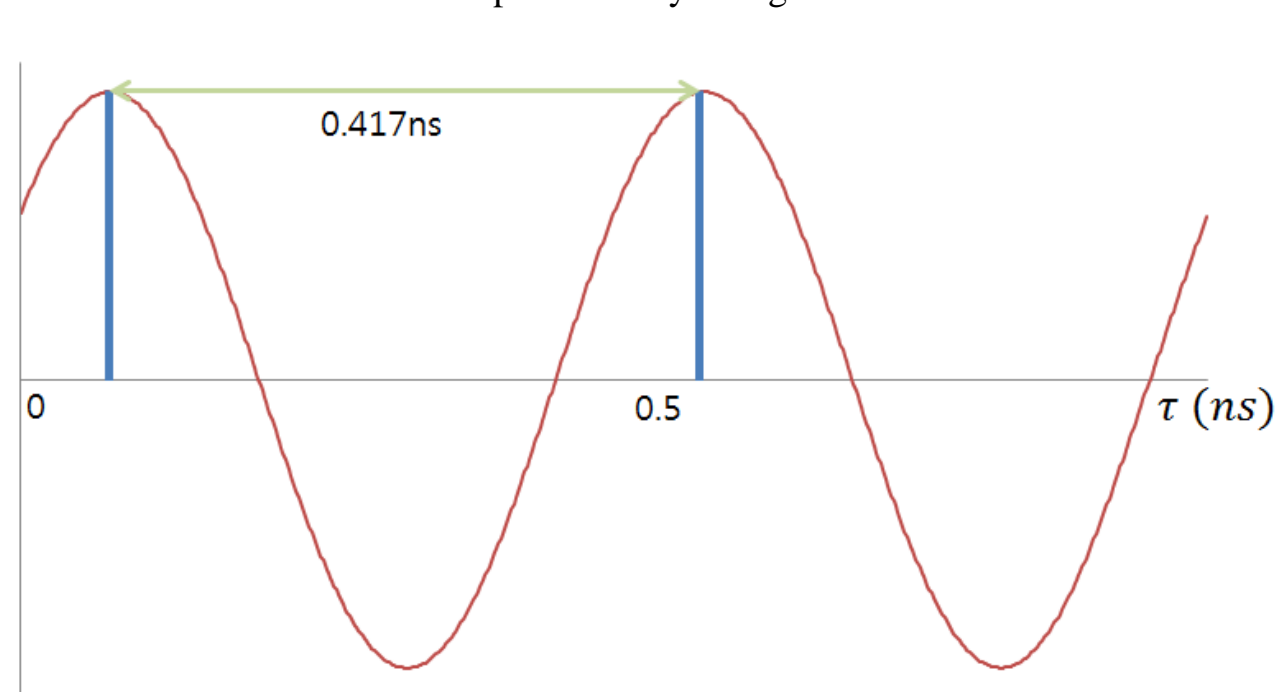

Figure 4. 2.4GHz Frequency Form

Figure 4 shows the $2.4 \mathrm{GHz}$ frequency on Wi-Fi. ToF is calculated using a phase difference of a frequency. But Wi-Fi based positioning is hard to get time of flight. A system that can accurately measure the absolute time of flight is needed. Some ultra wideband radios that span multiple Ghz can be used. A Wi-Fi radio can emulate a wideband multiple-GHz radio. Packets on multiple Wi-Fi bands and stitches their information together for giving the illusion of a wideband radio. Stitching information across such packets need to overcome three challenges. Resolving phase offsets, Eliminating packet detection delay, combating multipath are required[16].

A cycle of the frequency at $2.4 \mathrm{GHz}$ is $0.417 \mathrm{~ns}$. The distance between the transceiver and receiver can be obtained by using the TOF. By multiplying the ToF with the speed of light, a mobile client device can compute the distance between the transmitter and each of the receiver[16].

$\tau=-\frac{\angle h}{2 \pi f} \bmod \frac{1}{f} \quad(\tau$ : time of flight $)$

Formula 2 is used for getting the ToF with the Chinese remainder theorem in several channels. 


\section{Distance Decision based on RSSI}

Obtaining the position of an object based on a Wi-Fi signal depends on the accuracy of the distance corresponding to the RSSI values. As shown before, RSSI values change irregularly. To get more reliable signal values, the number of sampling data must be large. To decrease errors, more signal sampling data are needed.

Table 4. Calculated Distance Error According to the Number of Samples at $1 \mathrm{~m}$ from the Signal Transmitter

\subsection{RSSI Signal Characteristics in the $5 \mathrm{GHz}$}

\begin{tabular}{|c|c|}
\hline Sampling number & Error distance \\
\hline 1 time & $3.01 \mathrm{~m}$ \\
\hline 4 times & $1.53 \mathrm{~m}$ \\
\hline 6 times & $1.10 \mathrm{~m}$ \\
\hline 8 times & $1.12 \mathrm{~m}$ \\
\hline
\end{tabular}

\section{Table 5. RSSI Values at a 1m Distance between Tranismitter and Receiver}

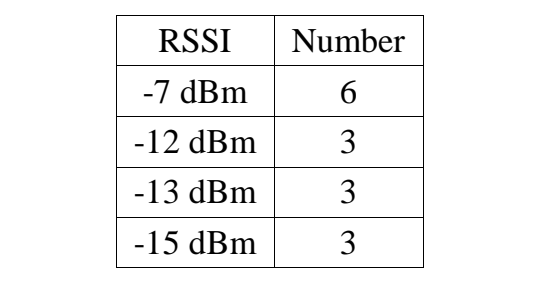

Table 5 shows 15 samples taken at a $1 \mathrm{~m}$ distance between the transmitter of an AP and the receivers of mobile objects in an indoor space. RSSI signal strength at $1 \mathrm{~m}$ between the transmitter and the receiver shows a variety of data ranging from $-7 \mathrm{dBm}$ to $-15 \mathrm{dBm}[9]$. Another AP and mobile object were used for sampling, and almost similar results were obtained. To get nore accurate results by using Formula (1), deciding the correct A (signal strength at $1 \mathrm{~m}$ ) is more important. But, as shown in Table 3 , the RSSI values at $1 \mathrm{~m}$ that were used as A in Formula (1) have some errors.

Therefore, getting reliable, stable signal values at $1 \mathrm{~m}$ for Formula (1) must be considered. More accurate RSSI values at $1 \mathrm{~m}$ could be estimated using $2 \mathrm{~m}$ to $5 \mathrm{~m}$ signal values. The effects of variations of A (RSSI values at $1 \mathrm{~m}$ ) and $\mathrm{n}$ (propagation loss) must be studied to calculate correct distances by using RSSI signals $[5,12]$.

Table 6. Signal Strength Differences in Distances

\begin{tabular}{|c|c|c|c|c|c|c|}
\multirow{2}{*}{ Distance } & \multicolumn{2}{|c|}{ Difference A -5dBm } & \multirow{2}{*}{ Difference } & \multicolumn{2}{|c|}{ Difference A -3dBm } & \multirow{2}{*}{ Difference } \\
\cline { 2 - 3 } & CASE1 & CASE2 & & CASE1 & CASE2 & \multirow{2}{*}{} \\
\hline $1 \mathrm{~m}$ & $-15 \mathrm{dBm}$ & $-20 \mathrm{dBm}$ & 5 & $-15 \mathrm{dBm}$ & $-18 \mathrm{dBm}$ & 3 \\
\hline $2 \mathrm{~m}$ & $-24 \mathrm{dBm}$ & $-29 \mathrm{dBm}$ & 5 & $-24 \mathrm{dBm}$ & $-27 \mathrm{dBm}$ & 3 \\
\hline $3 \mathrm{~m}$ & $-29 \mathrm{dBm}$ & $-34 \mathrm{dBm}$ & 5 & $-29 \mathrm{dBm}$ & $-32 \mathrm{dBm}$ & 3 \\
\hline $4 \mathrm{~m}$ & $-33 \mathrm{dBm}$ & $-38 \mathrm{dBm}$ & 5 & $-33 \mathrm{dBm}$ & $-36 \mathrm{dBm}$ & 3 \\
\hline $5 \mathrm{~m}$ & $-36 \mathrm{dBm}$ & $-41 \mathrm{dBm}$ & 5 & $-36 \mathrm{dBm}$ & $-39 \mathrm{dBm}$ & 3 \\
\hline
\end{tabular}

Table 6 shows two cases. CASE 1 and CASE 2 both use a value of 3.0 for $n$ (propagation loss). In CASE 1, the signal at $1 \mathrm{~m}$ is $-15 \mathrm{dBm}$, and in CASE 2 the signal at $1 \mathrm{~m}$ is $-20 \mathrm{dBm}$; the difference in the RSSI values for CASE 1 and CASE 2 is $5 \mathrm{dBm}$. The difference in RSSI values depending on distance in CASE 1 and CASE 2 show the same 
$-5 \mathrm{dBm}$ from $1 \mathrm{~m}$ to $5 \mathrm{~m}$. The values from $2 \mathrm{~m}$ to $5 \mathrm{~m}$ are calculated using Formula (1) based on $1 \mathrm{~m}$. The regular difference characteristic in Table 6 can be used to decide $\mathrm{n}$. A (the RSSI value at $1 \mathrm{~m}$ from the AP) can be decided using $\mathrm{n}$ with the given RSSI values from $2 \mathrm{~m}$ to $3 \mathrm{~m}$ under any circumstance.

\subsection{Fluctuation of the RSSI Values by Antenna Directions}

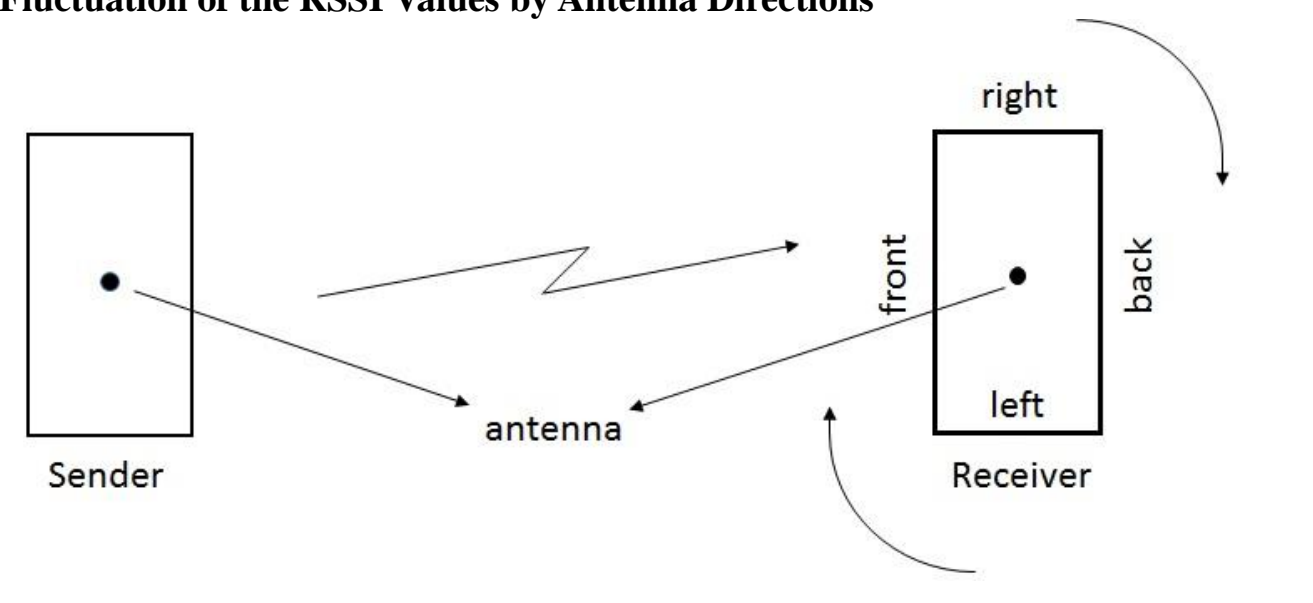

Figure 5. Four Directions of the Receiver

Figure 5 shows the direction of the receiver antenna in relation to the sender.

Table 7 shows the median value from 50 sample data) The RSSI values of a receiver for various antenna directions at a $2 \mathrm{~m}$ distance are shown:.[18]

\section{Table 7. RSSI Values According to the Direction of the Antenna}

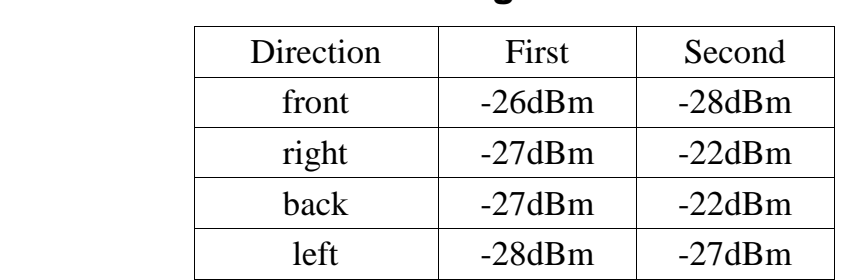

The strength of a signal changes depending on time and receiver antenna direction for the same position. To get more reliable data, some of the extraordinarily weak RSSI values are eliminated, and then an average of the remaining data is used.

\subsection{Errors Depend on Propagation Distance}

Signal strength of sampled data from farther away than $5 \mathrm{~m}$ is almost unchanged in Table 1. It is hard to distinguish distance using signals farther than $5 \mathrm{~m}$ between AP and mobile objects based on the experimental RSSI values.

Therefore, the distance between each AP and mobile object must be determined from within $5 \mathrm{~m}$ for location tracking. A $6 \mathrm{~m} \times 6 \mathrm{~m}$ square topology is considered an effective placement in the experimental area for location tracking. To use triangulation, at least three meaningful AP signals are needed. The distance from an AP to the mobile object must be less than $5 \mathrm{~m}$. At any position in the square, the distance between the mobile object and three APs is within 5m. One considerable arrangement of APs is the $6 \mathrm{~m} \times 6 \mathrm{~m}$ square topology shown in Figure 6 [6,7]. 


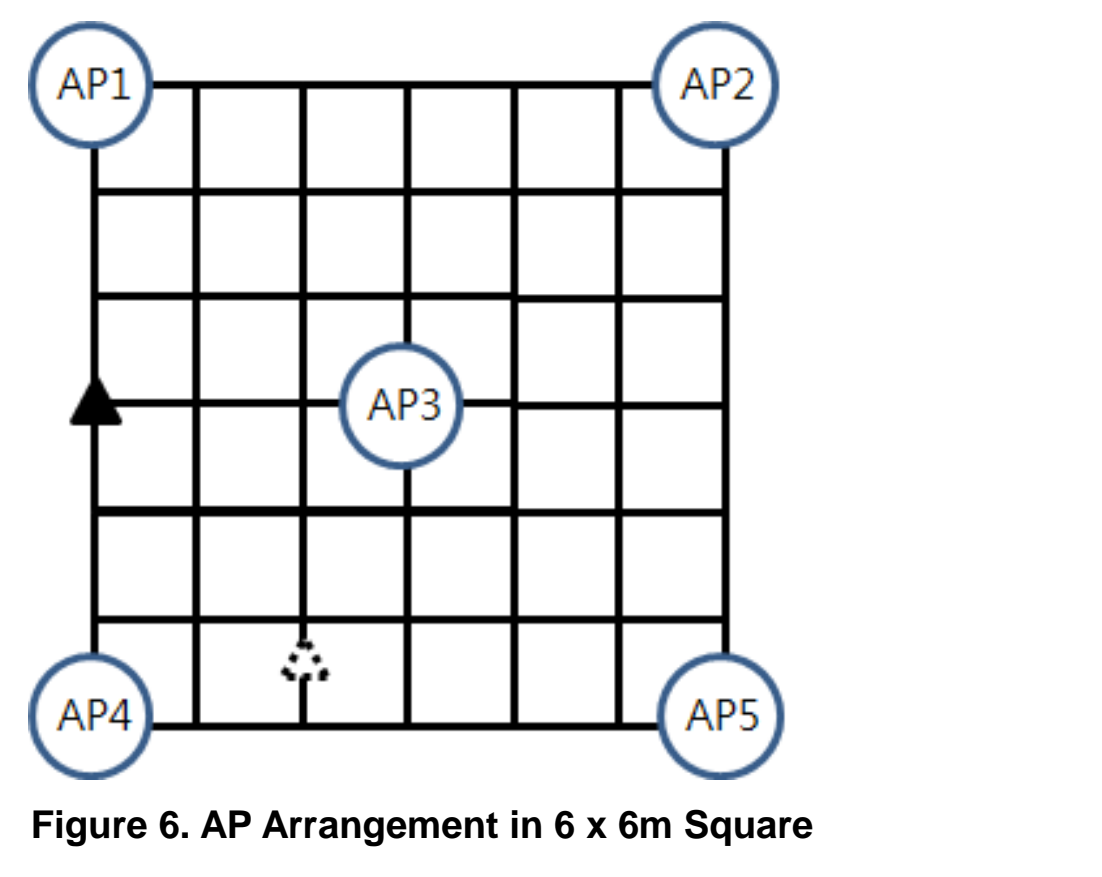

The measured signals at the black triangle position in Figure 6 are shown in Table 8. The chosen three closer APs based on the signals should be AP1, AP3, and AP4 [10,18].

\section{Table 8. Signál Strength cáculation for each AP}

\begin{tabular}{|c|c|c|c|c|}
\hline AP1 & AP2 & $\bullet$ AP3 & AP4 & AP5 \\
\hline $4.68 \mathrm{~m}$ & $6.62 \mathrm{~m}$ & $\mathbf{2 . 4 5 m}$ & $1.61 \mathrm{~m}$ & $4.43 \mathrm{~m}$ \\
\hline
\end{tabular}

But some abnomar situations are observed. Table 8 shows the results of calculating distance using Formula (1) for RSSI signals. The errors in the signals give incorrect results.

In Figure 6, APs closer to the mobile object, shown as a black triangle, are AP1, AP3, and AP4, but the calculation results show that AP3, AP4, and AP5 are closer.

This may lead o mistaken position for the mobile object, placing it at the triangle with square dotted lines. Other types of topology must be studied.

\section{Experiment Environments for Location Decision}

\section{Positioning using RSSI from Wi-Fi}

An experiment was carried out based on the Figure 6 topology. The five APs are Iptime's dual band Wi-Fi AP Multi, and the receiver of the mobile object is Iptime's A2000UA.

In the experiment, signal reflectance by the floor is observed. Therefore, all of the devices were placed and measured at a height of $2 \mathrm{~m}$ above the floor [13]. 
Table 9. RSSI Values According to Real Distances from each AP, based on the Figure 6 Topology

\begin{tabular}{|l|l|l|l|}
\hline & $2 \mathrm{~m}$ & $3 \mathrm{~m}$ & $4 \mathrm{~m}$ \\
\hline AP1 & -28.5 & -31.5 & -36 \\
\hline AP2 & -26.5 & -31 & -32.7 \\
\hline AP3 & -27 & -31 & -33.5 \\
\hline AP4 & -26.5 & -29 & -32.5 \\
\hline AP5 & -28 & -32.5 & -36 \\
\hline
\end{tabular}

Table 9 shows the RSSI values corresponding to the distance. Using the results of table 9 , the values of $\mathrm{n}$ and $\mathrm{A}$ at $1 \mathrm{~m}$ are estimated.

Table 10. Values for $\mathrm{n}$ and $\mathrm{A}$ at $\mathbf{1 m}$ from the APs

\begin{tabular}{|c|c|c|c|c|}
\hline \multicolumn{5}{|c|}{ Propagation Loss } \\
\hline AP1 & AP2 & AP3 & AP4 & AP5 \\
\hline 2.0 & 2.0 & 2.0 & 2.0 & 2.0 \\
\hline \multicolumn{5}{|c|}{ RSSI value } \\
\hline AP1 & AP2 & AP3 & AP4 & AP5 \\
\hline-22 & -20 & -21 & -20 & -22 \\
\hline
\end{tabular}

Table 11. Measured bistance from each AP

\begin{tabular}{|l|l|l|l|l|l|}
\hline & AP1 & AP2 & AP3 & AP4 & AP5 \\
\hline $\begin{array}{l}\text { Measured } \\
\text { distances }\end{array}$ & $12.59 \mathrm{~m}$ & $15.85 \mathrm{~m}$ & $\mathbf{1} 1.78 \mathrm{~m}$ & $14.13 \mathrm{~m}$ & $3.98 \mathrm{~m}$ \\
\hline
\end{tabular}

Table 11 shows the results of calculating distance by using the RSSI values of the APs at the measurement positions. The quadrant of the topology is selected by the smallest value of the measured distances. The three selected APs are AP3, AP5, and AP1. In this case, the position is placed between quadrants 3 and 4 . So a large error is observed.

Table 12. Quadrants and Error Rate

\begin{tabular}{|c|l|l|}
\hline & Quadrant & Error rate \\
\hline Reallocation & 4 & $0 \mathrm{~m}$ \\
\hline Assumed location & 4 & $0.44 \mathrm{~m}$ \\
\hline Caleualted location & 3 or 4 & $1.65 \mathrm{~m}$ \\
\hline
\end{tabular}

Table 2 shows when a decision for the quadrant is wrong; then, the error rate become brgger at the calculated position. If the same quadrant is decided as an estimated position, then the calculated position may have less of a distance error. In the experiment, because of the different quadrants of the measurement location, the calculated position has larger errors.

\subsection{Positioning using Laser Sensor and Compass Sensor}

An experiment was carried out based on the layout in Figure 7. The scale of the room layout is provided. The compass sensor used was NTrex's NT-Mag6352, and the laser sensor was LULSED LIGHT's LIDAR-Lite. To track the position of mobile nodes, a geomagnetic sensor is first used to get a bearing angle, and then, a laser sensor gets distance in one direction. At least two directions for distance may be needed to decide a position, compared with the room layout information. 


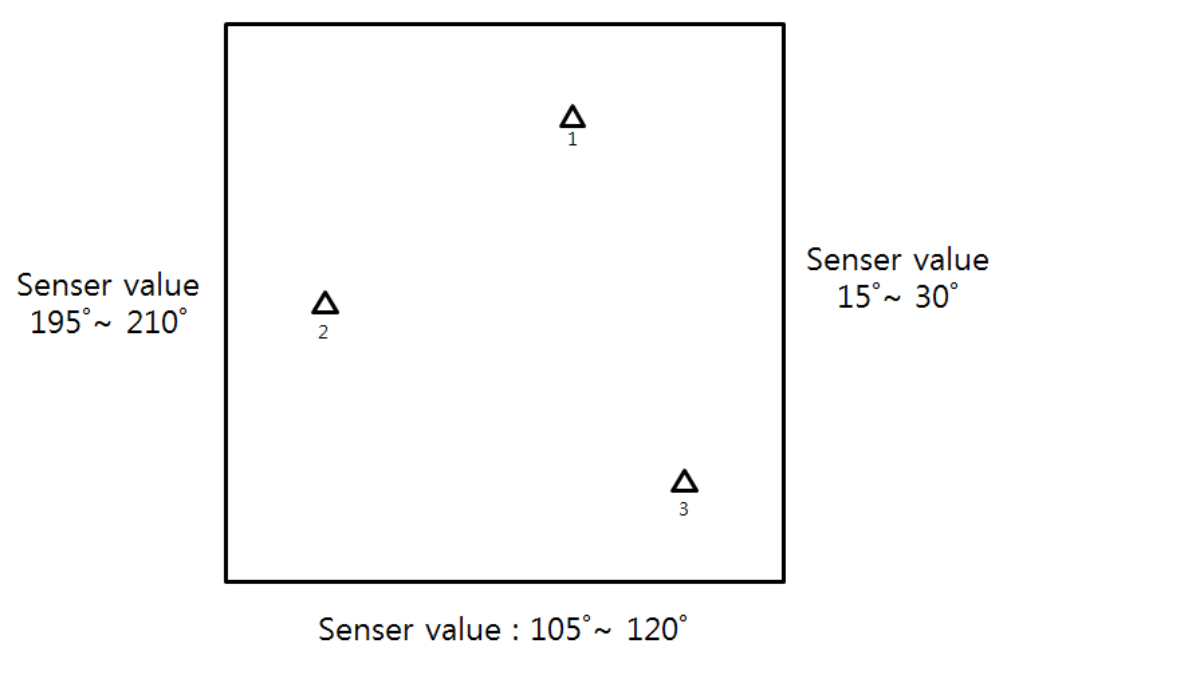

Figure 7. Three Arbitrary Positions in a $6 \mathrm{~m} \times 6 \mathrm{~m}$ Layout

Figure 7 shows three arbitrary mobile objecpositions used in the experiments.

Table 13. Bearing Value and Distance Value at the Position

\begin{tabular}{|l|lll|l|l|}
\hline Location & $\begin{array}{l}\text { Bearing } \\
\text { value }\end{array}$ & bistance & $\begin{array}{l}\text { Bearing } \\
\text { value }\end{array}$ & Distance \\
\hline 1 & $292^{\circ}$ & 1.026 & $202^{\circ}$ & $3.56 \mathrm{~m}$ \\
\hline 2 & $505^{\circ}$ & $5.03 \mathrm{~m}$ & $205^{\circ}$ & $1.01 \mathrm{~m}$ \\
\hline 3 & $288^{\circ}$ & $5.07 \mathrm{M}$ & $198^{\circ}$ & $5.03 \mathrm{~m}$ \\
\hline
\end{tabular}

Table 13 show some results that were measured as the distance values and the bearing angles at the three arbitiary positions. The distance values were measured several times from the target wall to the mobile object by rotating the object in $10^{\circ}$ directions. The final distance in azimuthris determined by selecting the shortest value of the measured values.

Table 14. Positioning Error between Actual Position and the

\begin{tabular}{|c|c|c|c|c|c|}
\multicolumn{5}{c|}{ Measured Position } \\
\multirow{2}{*}{ Location } & \multicolumn{2}{|c|}{ Actual distance } & \multicolumn{2}{c|}{$\begin{array}{c}\text { Measurement } \\
\text { distance }\end{array}$} & \multirow{2}{*}{ Error rate } \\
\cline { 2 - 6 } & Width & Height & Width & Height & \\
\hline 1 & $3.5 \mathrm{~m}$ & $1 \mathrm{~m}$ & $3.56 \mathrm{~m}$ & $1.02 \mathrm{~m}$ & $6.32 \mathrm{~cm}$ \\
\hline 2 & $1 \mathrm{~m}$ & $3 \mathrm{~m}$ & $1.01 \mathrm{~m}$ & $3.03 \mathrm{~m}$ & $3.16 \mathrm{~cm}$ \\
\hline 3 & $5 \mathrm{~m}$ & $5 \mathrm{~m}$ & $5.03 \mathrm{~m}$ & $5.07 \mathrm{~m}$ & $7.61 \mathrm{~cm}$ \\
\hline
\end{tabular}

Table 14 shows positioning errors between actual positions and the measured positions.

The positioning error by using the laser sensor and the geomagnetic sensor is very low. The error is less than $10 \mathrm{~cm}$. 


\section{Proposed Hybrid Indoor Positioning System}

A hybrid method for tracking indoor location is proposed. Figure 8 shows an example environment. The followings are prerequisites. A blueprint that shows the precise scales and bearing angles of the room layout is needed. Some Wi-Fi APs are located in designated positions. The mobile object decides roughly a room by Wi-Fi signals using the layout, and then an accurate position is decided using a compass sensor and a laser sensor by scaling the distances from the wall. By accumulating the real-time positioning data, the system can work as an indoor navigation or security system.

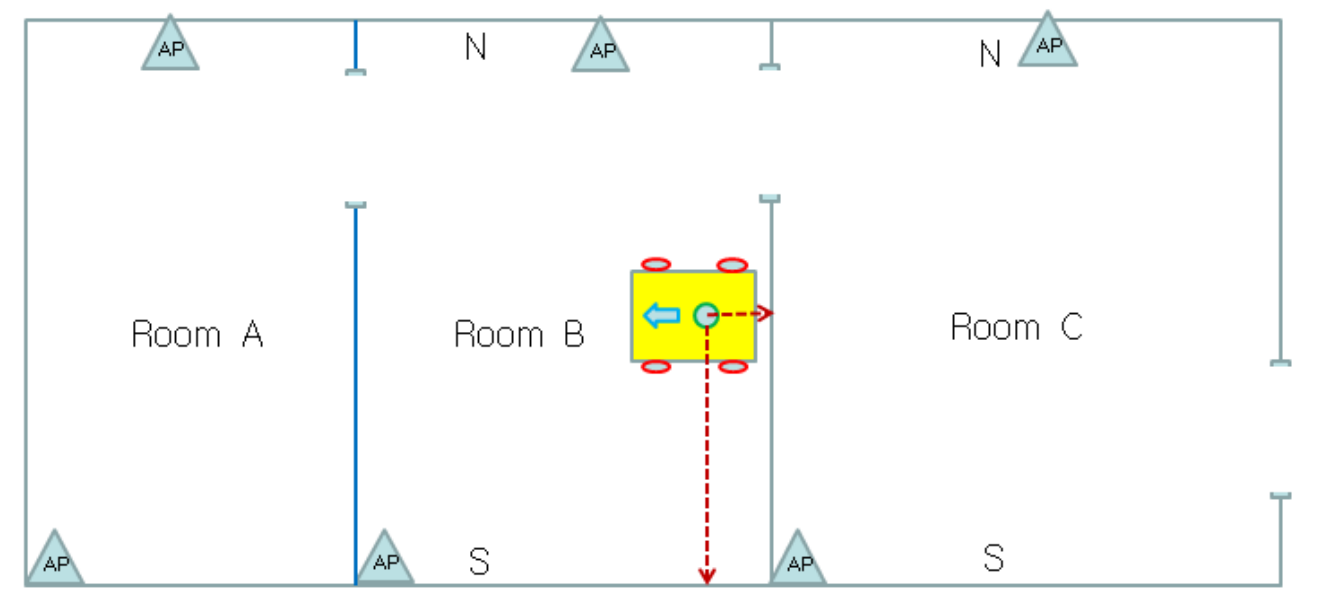

Figure 8. An Example of the Hybrid Indoor Positioning

\section{Conclusions}

Indoor location tracking by using Wu-Fi RSSI signal strengths is studied. The signal strength of wireless is not always corresponding, especially longer than $5 \mathrm{~m}$, to the propagation distance. In orden to increase the accuracy of the location estimation, triangle quadrants of the rectangle is introduced to predict precisely where the mobile object is located.

In order to reduce the position errors and to avoid selection of wrong quadrants, some additional methods are needed. Some of candidate additional techniques are considered to improve accuracy. But it has also errors due to the surrounding environment. The RSSI based method is not sufficient to improve positioning accuracy. The need for introducing ToF(Time of Firght) to replace the RSSI in the Wi-Fi environment is increased.

The effect of sampling frequency in an arbitrary position is additionally studied. To inerease correctness for deciding correct location, some epochal positioning methods for location of mobile object must be studied. As a method, laser sensor and geomagnetic sensor are introduced.

By combining Wi-Fi and other sensors like laser sensor and geomagnetic sensor, more efficient and accurate indoor positioning can be achieved.

\section{Acknowledgements}

This work was funded by the BK21+ program of the National Research Foundation of Korea (NRF). This research was also supported by The Leading Human Resource Training

Program of the Regional Neo industry through the National Research Foundation of Korea (NRF) funded by the Ministry of Science, ICT and Future Planning (216C000360). 


\section{References}

[1] "Wireless LAN-Based LBS Services", Hakyong KIM, apr, 2006

[2] Tai-Gil Kwon, Jin-Woong Cho, Seung Ok Lim, Jang Yeon Lee, Hyeon Seok Lee, Yun Jae Won "A Study on Distance Measurement using CSS and RSSI in WPAN"

[3] Chipcon formula, http://www.ti.com

[4] Patric Jensfelt , Patric Jensfelt , "Laser Based Position Acquisition and Tracking in an Indoor Environment" Dec. 1998

[5] C. Praneeth Kumar, Ravi Poovaiah, Ajanta Sen, Priya Ganadas"Single Access Point-based Indoor Localization Technique for Augmented Reality Gaming for Children" Feb. 2014

[6] HyunJin Park, GyeungnWook Min, ,HyeonCheol Zin, YoungRak Kim, ChongGun Kim, “An efficient AP allocation for position tracking lased on Wi-Fi” Oct. 2015

[7] Jong-Hoon, Sohn, Gi-Hyun Hwang, "Development of Position Awareness Algorithm Using Improved Trilateration Measurement Method" Mar. 2013

[8] Atreyi Bose and Chuan Heng Foh, "A Practical Path Loss Model For Indoor WiFi Positioning Enhancement" Dec. 2007

[9] Dae-young Lee, Young-heung Kang "Smart Phone Sensor-Based Indoor Location Tracking System for Improving the Location Error of the Radio Environment" Feb. 2015

[10] Gaoyang Shan, Byeong-hun Park, Song-hyeon Nam, Bosung Kim, Byeong-hee Roh, Young-Bae Ko "A 3-Dimensional Triangulation Scheme To Improve The Accuracy of Indoor Localization for IoT Services" Aug. 2015

[11] J. Machaj, P. Brida , R. Piché ,"Rank Based Fingerprinting Algorithm for Indoor Positioning" Sept. 2011

[12] Qian Dong, Waltenegus Dargie "Evaluation of the Reliability of RSSI for Indoor Localization" Aug. 2012

[13] Hung-Huan Liu, Yu-Non Yang, "WiFi-Based Indopr Positioning for Multi-Floor Environment" Nov. 2011

[14] Apostolia Papapostolou, Hakima Chaouchi, 'NAFE: Wireless Indoor Positioning Based on Fingerprint Evaluation" May. 2009

[15] Cheng Bo, Guobin Shen, Chunshui Zhao, Liqun L1, and Feng Zhao, "Magicol: Indoor Localization Using Pervasive Magnetic Field ápd Opportunistic W'íi Sensing” Jun 2015

[16] Deepak Vasisht, Swarun Kumar, Dina Katabi , "Decimeter-Level Localization with a

Single WiFi Access Point" MAR. 2016

[17] Yang, Jeong-Woo; An, Gae--1; Kim, Shin-Hyo; Chung, Byung-Ho; Kim, Tae-Yeon; Pyun, Ki-Hyun; Cho, Gi-HwanA "Distance Estimation Scheme Based on WLAN RF Properties for Localization of Mobile Terminals" 2014

[18] HyunJin Park, JaeMin Hong, WenJuñ Zhu, DongHyun Lee, Hyeoncheol Zin, YoungRak Kim, ChongGun Kim Analysis of selected indoor location APs for in Wi-Fi environments" 2016

[19] Journal of Fluid Mechanics "The Earth s magnetic field: Its history, origin and planetary perspective" July 1985

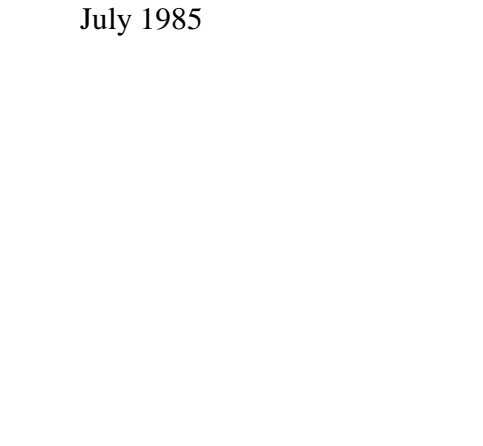

